\title{
Ciência e Tecnologia
}

Para o Desenvolvimento

Ambiental, Cultural

e Socioeconômico

Leinig Antonio Perazolli

(organizador) 


\section{Ciência e Tecnologia}

Para o Desenvolvimento

Ambiental, Cultural

e Socioeconômico

Leinig Antonio Perazolli

(organizador) 


\section{1 by Editora Artemis \\ Copyright (C) Editora Artemis \\ Copyright do Texto (C) 2021 Os autores \\ Copyright da Edição (C) 2021 Editora Artemis}

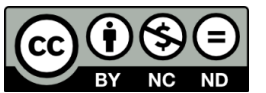

O conteúdo deste livro está licenciado sob uma Licença de Atribuição Creative Commons Atribuição-Não-Comercial NãoDerivativos 4.0 Internacional (CC BY-NC-ND 4.0). Direitos para esta edição cedidos à Editora Artemis pelos autores. Permitido o download da obra e o compartilhamento, desde que sejam atribuídos créditos aos autores, e sem a possibilidade de alterá-la de nenhuma forma ou utilizá-la para fins comerciais.

A responsabilidade pelo conteúdo dos artigos e seus dados, em sua forma, correção e confiabilidade é exclusiva dos autores. A Editora Artemis, em seu compromisso de manter e aperfeiçoar a qualidade e confiabilidade dos trabalhos que publica, conduz a avaliação cega pelos pares de todos manuscritos publicados, com base em critérios de neutralidade e imparcialidade acadêmica.

$\begin{array}{ll}\text { Editora Chefe } & \text { Profa Dra Antonella Carvalho de Oliveira } \\ \text { Editora Executiva } & \text { M. a Viviane Carvalho Mocellin } \\ \text { Direção de Arte } & \text { M. a Bruna Bejarano } \\ \text { Diagramação } & \text { Elisangela Abreu } \\ \text { Organizador } & \text { Prof. Dr. Leinig Antonio Perazolli } \\ \text { Imagem da Capa } & \text { peacestock/123RF } \\ \text { Bibliotecário } & \text { Maurício Amormino Júnior - CRB6/2422 }\end{array}$

\section{Conselho Editorial}

Prof.a Dr.a Ada Esther Portero Ricol, Universidad Tecnológica de La Habana "José Antonio Echeverría", Cuba

Prof. Dr. Adalberto de Paula Paranhos, Universidade Federal de Uberlândia

Prof.a Dr.a Amanda Ramalho de Freitas Brito, Universidade Federal da Paraíba

Prof. a Dr.a Ana Clara Monteverde, Universidad de Buenos Aires, Argentina

Prof. Dr. Ángel Mujica Sánchez, Universidad Nacional del Altiplano, Peru

Prof.a Dr.a Angela Ester Mallmann Centenaro, Universidade do Estado de Mato Grosso

Prof.a Dr.a Begoña Blandón González, Universidad de Sevilla, Espanha

Prof. a Dr.a Carmen Pimentel, Universidade Federal Rural do Rio de Janeiro

Prof. a Dr.a Catarina Castro, Universidade Nova de Lisboa, Portugal

Prof.a Dr.a Cláudia Padovesi Fonseca, Universidade de Brasília-DF

Prof.a Dr.a Cláudia Neves, Universidade Aberta de Portugal

Prof. Dr. Cleberton Correia Santos, Universidade Federal da Grande Dourados

Prof. Dr. David García-Martul, Universidad Rey Juan Carlos de Madrid, Espanha

Prof.a Dr.a Deuzimar Costa Serra, Universidade Estadual do Maranhão

Prof. a Dr.a Eduarda Maria Rocha Teles de Castro Coelho, Universidade de Trás-os-Montes e Alto Douro, Portugal

Prof. Dr. Eduardo Eugênio Spers, Universidade de São Paulo

Prof. Dr. Eloi Martins Senhoras, Universidade Federal de Roraima

Prof.a Dr.a Elvira Laura Hernández Carballido, Universidad Autónoma del Estado de Hidalgo, México

Prof. a Dr.a Emilas Darlene Carmen Lebus, Universidad Nacional del Nordeste/ Universidad Tecnológica Nacional, Argentina

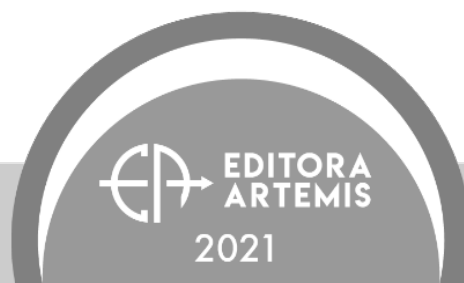


Prof. a Dr. a Erla Mariela Morales Morgado, Universidad de Salamanca, Espanha

Prof. Dr. Ernesto Cristina, Universidad de la República, Uruguay

Prof. Dr. Ernesto Ramírez-Briones, Universidad de Guadalajara, México

Prof. Dr. Gabriel Díaz Cobos, Universitat de Barcelona, Espanha

Prof. Dr. Geoffroy Roger Pointer Malpass, Universidade Federal do Triângulo Mineiro

Prof.a Dr.a Gladys Esther Leoz, Universidad Nacional de San Luis, Argentina

Prof.a Dr.a Glória Beatriz Álvarez, Universidad de Buenos Aires, Argentina

Prof. Dr. Gonçalo Poeta Fernandes, Instituto Politécnido da Guarda, Portugal

Prof. Dr. Gustavo Adolfo Juarez, Universidad Nacional de Catamarca, Argentina

Prof.a Dr.a Iara Lúcia Tescarollo Dias, Universidade São Francisco

Prof. a Dr.a Isabel del Rosario Chiyon Carrasco, Universidad de Piura, Peru

Prof.a Dr.a Isabel Yohena, Universidad de Buenos Aires, Argentina

Prof. Dr. Ivan Amaro, Universidade do Estado do Rio de Janeiro

Prof. Dr. Iván Ramon Sánchez Soto, Universidad del Bío-Bío, Chile

Prof. a Dr. a Ivânia Maria Carneiro Vieira, Universidade Federal do Amazonas

Prof. Me. Javier Antonio Albornoz, University of Miami and Miami Dade College, USA

Prof. Dr. Jesús Montero Martínez, Universidad de Castilla - La Mancha, Espanha

Prof. Dr. João Manuel Pereira Ramalho Serrano, Universidade de Évora, Portugal

Prof. Dr. Joaquim Júlio Almeida Júnior, UniFIMES - Centro Universitário de Mineiros

Prof. Dr. Juan Carlos Mosquera Feijoo, Universidad Politécnica de Madrid, Espanha

Prof. Dr. Juan Diego Parra Valencia, Instituto Tecnológico Metropolitano de Medellín, Colômbia

Prof. Dr. Júlio César Ribeiro, Universidade Federal Rural do Rio de Janeiro

Prof. Dr. Leinig Antonio Perazolli, Universidade Estadual Paulista

Prof.a Dr.a Lívia do Carmo, Universidade Federal de Goiás

Prof. a Dr.a Luciane Spanhol Bordignon, Universidade de Passo Fundo

Prof. Dr. Luis Vicente Amador Muñoz, Universidad Pablo de Olavide, Espanha

Prof. a Dr.a Macarena Esteban Ibáñez, Universidad Pablo de Olavide, Espanha

Prof. Dr. Manuel Ramiro Rodriguez, Universidad Santiago de Compostela, Espanha

Prof. Dr. Marcos Augusto de Lima Nobre, Universidade Estadual Paulista

Prof. Dr. Marcos Vinicius Meiado, Universidade Federal de Sergipe

Prof.a Dr. a Mar Garrido Román, Universidad de Granada, Espanha

Prof. a Dr.a Margarida Márcia Fernandes Lima, Universidade Federal de Ouro Preto

Prof. a Dr. a Maria Aparecida José de Oliveira, Universidade Federal da Bahia

Prof.a Dr. a Maria do Céu Caetano, Universidade Nova de Lisboa, Portugal

Prof. a Dr. a Maria do Socorro Saraiva Pinheiro, Universidade Federal do Maranhão

Prof.a Dr.a Maria Lúcia Pato, Instituto Politécnico de Viseu, Portugal

Prof. a Dr. a Maritza González Moreno, Universidad Tecnológica de La Habana "José Antonio Echeverría", Cuba

Prof.a Dr. a Mauriceia Silva de Paula Vieira, Universidade Federal de Lavras

Prof. a Dr. a Odara Horta Boscolo, Universidade Federal Fluminense 
Prof.a Dr.a Patrícia Vasconcelos Almeida, Universidade Federal de Lavras Prof. a Dr. a Paula Arcoverde Cavalcanti, Universidade do Estado da Bahia Prof. Dr. Rodrigo Marques de Almeida Guerra, Universidade Federal do Pará Prof. Dr. Saulo Cerqueira de Aguiar Soares, Universidade Federal do Piauí Prof. Dr. Sergio Bitencourt Araújo Barros, Universidade Federal do Piauí Prof. Dr. Sérgio Luiz do Amaral Moretti, Universidade Federal de Uberlândia Prof. a Dr. a Silvia Inés del Valle Navarro, Universidad Nacional de Catamarca, Argentina Prof. a Dr. - Teresa Cardoso, Universidade Aberta de Portugal Prof. a Dr. a Teresa Monteiro Seixas, Universidade do Porto, Portugal

Prof. Dr. Turpo Gebera Osbaldo Washington, Universidad Nacional de San Agustín de Arequipa, Peru Prof. Dr. Valter Machado da Fonseca, Universidade Federal de Viçosa Prof. a Dr.a Vanessa Bordin Viera, Universidade Federal de Campina Grande Prof. a Dr. a Vera Lúcia Vasilévski dos Santos Araújo, Universidade Tecnológica Federal do Paraná Prof. Dr. Wilson Noé Garcés Aguilar, Corporación Universitaria Autónoma del Cauca, Colômbia

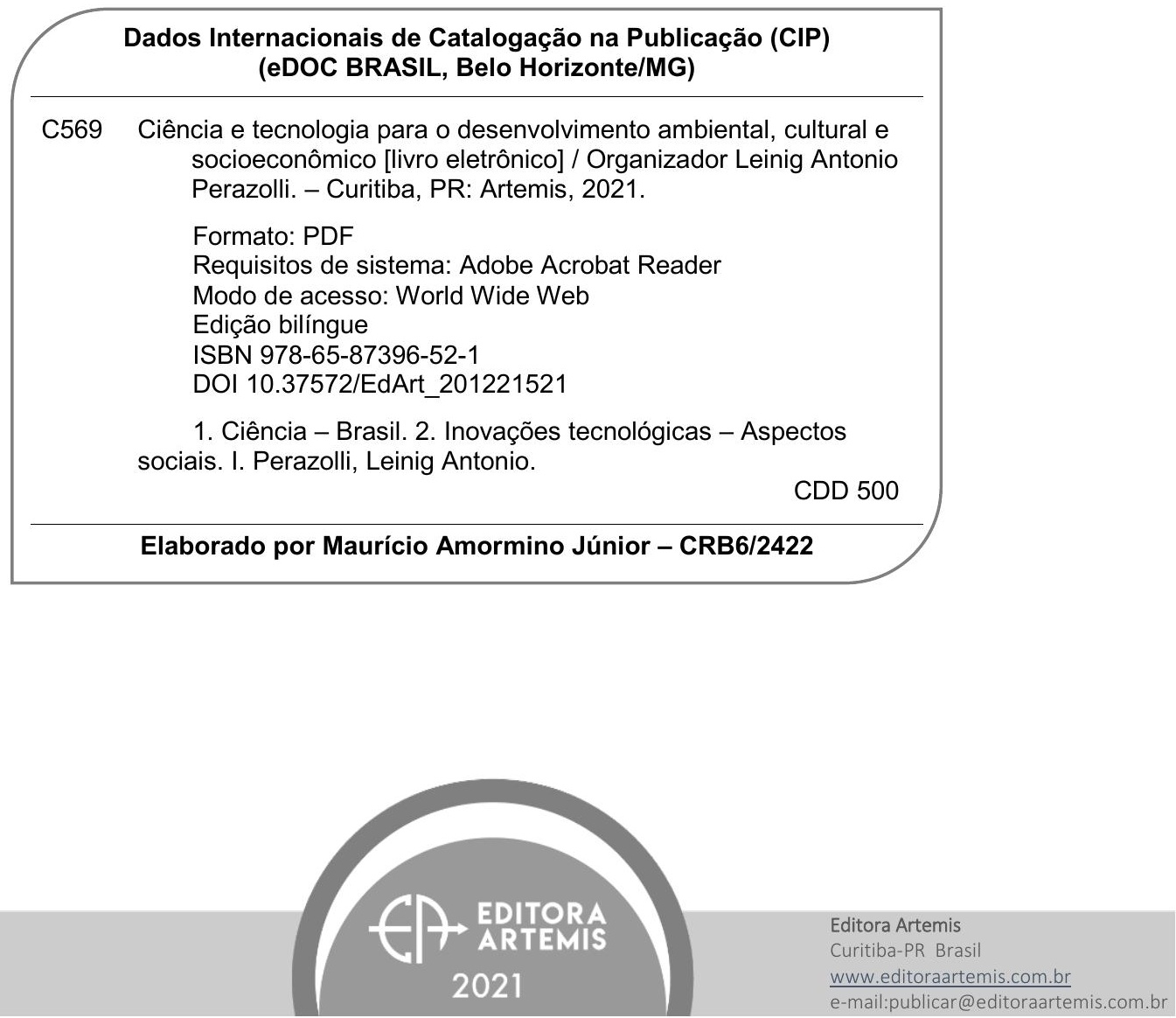




\section{APRESENTAÇÃO}

\section{A publicação intitulada "Ciência e Tecnologia para o Desenvolvimento}

Ambiental, Cultural e Socioeconômico" faz uma coletânea de resultados científicos, em diferentes áreas do conhecimento, exemplificando um modelo para a abordagem dos problemas relacionados ao desenvolvimento ambiental, cultural e socioeconômico da sociedade atual.

A obra se justifica ao apresentar caminhos para se atingir soluções positivas frente às grandes e variadas dificuldades que estamos observando nas últimas décadas, decorrentes de ações comerciais, do desejo de consumo e ao fato que as fontes são finitas, porém os desejos humanos não. Estas ações comerciais levam à destruição ambiental, massificação cultural e a problemas socioeconômicos devido à diferença de renda e ao aumento da frequência de desastres ambientais, os quais geram grandes prejuízos financeiros e humanos.

A obra se inicia relatando o estado da arte sobre o consumo ético, avança para a descrição da vulnerabilidade e do emprego sustentável de ecossistemas. Destaca a função dos processos de educação, peça fundamental para a evolução sustentável de qualquer sociedade e a importância da interrelação entre os municípios de diferentes países para a busca de objetivos comuns.

$\mathrm{Na}$ continuidade temos exemplos de resultados científicos positivos para o uso de tecnologias em diferentes áreas do conhecimento, desde o uso de micro-organismos e sementes para a produção de óleos e energia, tratamentos e recuperação de resíduos de minerais e propostas científicas avançadas nas áreas de separação líquido-líquido, magneto eletrônica e varistores. A obra também ilustra as consequências das ações negativas praticadas pela ação humana. Cabe destacar que se estas ações não forem evitadas, corrigidas e/ou readequadas as consequências dos desastres ambientais, com reflexos negativos em todas as áreas, poderão se tornar irreversíveis em questão de décadas.

A importância deste livro reside ao indicar caminhos para fomentar o desenvolvimento ambiental, cultural e socioeconômico de forma sustentável.

Quero externar meus sinceros agradecimentos aos autores dos trabalhos científicos e à Editora Artemis, pela organização desta obra.

Prof. Dr. Leinig Antonio Perazolli UNESP - Instituto de Química de Araraquara/SP 
CAPÍTULO 1

ESTADO DEL ARTE SOBRE CONSUMO ÉTICO EN LA ÚLTIMA DÉCADA: REVISIÓN SISTEMÁTICA DE LA LITERATURA

Javier Solano

David Zaldumbide Peralvo

Delia García Vences

dø) https://doi.org/10.37572/EdArt_2012215211

CAPÍTULO 2. 18

VULNERABILIDAD DE LAS AGUAS SUBTERRÁNEAS A LA CONTAMINACIÓN POR NITRATO EN LA CUENCA DEL RÍO EBRO (ESPAÑA) MEDIANTE EL PROCEDIMIENTO LU-IV

Mercedes Arauzo Sánchez

María Valladolid Martín

Gema García González

doi)'https://doi.org/10.37572/EdArt_2012215212

CAPÍTULO 3. 36

ECOSISTEMAS DE EMPRENDIMIENTO, DE LO NACIONAL A LO REGIONAL, SANTANDER UNA APUESTA

Mónica María Pacheco Valderrama

Olga Cecilia Alarcón Vesga

do) https://doi.org/10.37572/EdArt_2012215213

CAPÍTULO 4 47

O ENSINO MÉDIO POLITÉCNICO E A REALIDADE TECNOLÓGICA VIVENCIADA PELOS ALUNOS E PROFESSORES EM DUAS ESCOLAS DE PELOTAS- RS

Elis Regina Madeira da Porciúncula

Marcos Antonio Anciuti

doi) https://doi.org/10.37572/EdArt_2012215214 
UNIVERSIDADES Y LA APROPIACIÓN SOCIAL DE LA CIENCIA. ANÁLISIS DEL SISTEMA DE CIENCIA Y TECNOLOGÍA DE CHILE

Juan Ramón Contreras González

doi)'https://doi.org/10.37572/EdArt_2012215215

CAPÍTULO 6 89

COMPARACIÓN DE LA NORMATIVA DE CONTROL INTERNO Y EXTERNO MUNICIPAL ENTRE ECUADOR Y ARGENTINA

Verónica Ponce

Carlos Albert Ferreira

José Townsend

doi) $h$ ttps://doi.org/10.37572/EdArt_2012215216

CAPÍTULO 7 103

EL USO DE LA BIOMASA DEL HONGO Aspergillus niger PARA LA ELIMINACIÓN DE METALES PESADOS DE AGUAS CONTAMINADAS

Ismael Acosta Rodríguez

Nancy Pacheco Castillo

Adriana Rodríguez Pérez

Juan Fernando Cárdenas González

Víctor Manuel Martínez Juárez

Francisco Navarro Castillo

Erika Enríquez Domínguez

Juana Tovar Oviedo

doi) https://doi.org/10.37572/EdArt_2012215217

CAPÍTULO 8. 114

INFLUENCIA DE LA ENVOLVENTE CON BAJOS NIVELES DE TRANSMITANCIA EN EL CONSUMO ENERGÉTICO DE VIVIENDAS EN CLIMAS CÁLIDOS

María Victoria Mercado

Celina Filippín

Gustavo Barea

doi) https://doi.org/10.37572/EdArt_2012215218 
ESTUDIO DE LA ACTIVIDAD ANTIMICROBIANA DE LA CUPRITA SINTETIZADA POR RUTA QUÍMICA

Orfelinda Avalo Cortez

David Pedro Martínez Aguilar

doi) $h$ https://doi.org/10.37572/EdArt_2012215219

CAPÍTULO 10. 147

ACEITE DE LA SEMILLA DE AGUACATE, UNA REVISIÓN DESDE SUS POTENCIALIDADES

Lina González Asías

Amelia Espitia Arrieta

Jennifer Lafont Mendoza

do) https://doi.org/10.37572/EdArt_20122152110

\section{CAPÍTULO 11}

ESTUDIOS QUÍMICOS REALIZADOS A LA SEMILLA DE Moringa oleifera Lam Y SU IMPACTO EN LA SALUD HUMANA: UNA REVISIÓN TEÓRICA

Jennifer Lafont Mendoza

William Negrete Humanez

Amelia Espitia Arrieta

do)'https://doi.org/10.37572/EdArt_20122152111

CAPÍTULO 12. 171

ZONAS DE INFLUENCIA GENERADAS POR PROPIEDADES FISICAS PARA LA CARACTERIZACION EN CAMPO DEL MATERIAL ROCOSO

Ernesto Patricio Feijoo Calle

Andrés Nicolás Aguirre Larriva

Bernardo Andrés Feijoo Guevara

do)'https://doi.org/10.37572/EdArt_20122152112

CAPÍTULO 13 186

CARACTERIZACIÓN Y FLOTACIÓN DE APATITA CONTENIDAS EN RELAVE DE HIERRO

Luis Valderrama

Mario Santander

Osvaldo Gómez 
Patricia Tapia

Patricio Muñoz

Bruno Zazzali

doi)https://doi.org/10.37572/EdArt_20122152113

\section{CAPÍTULO 14}

ESTUDO DE COMPÓSITOS COM MATRIZ DE ALUMÍNIO E RESÍDUOS DE MINÉRIO DE MANGANÊS POR SINTERIZAÇÃO AO AR NATURAL

Affonso Henrique Alves Ribeiro

Margarida Márcia Fernandes Lima

Rhelman Rossano Urzedo Queiroz

Rosa Malena Fernandes Lima

do) https://doi.org/10.37572/EdArt_20122152114

CAPÍTULO 15 214

EXTRAÇÃO LÍQUIDO-LÍQUIDO APLICADA AO PROCESSO DE REFINO DE ETANOL COMBUSTIVELL

Gabriel Manso Kozlowski Pitombeira

Leinig Antonio Perazolli

Elias de Souza Monteiro Filho

do)'https://doi.org/10.37572/EdArt_20122152115

CAPÍTULO 16.

EVIDENCING THE MAGNETOELECTRIC COUPLING IN BI1-XNDXFEO3 COMPOSITIONS THROUGH FERROIC CHARACTERIZATIONS

Anuar Jose Mincache

Lilian Felipe da Silva Tupan

Odair Gonçalves de Oliveira

Ivair Aparecido dos Santos

Luiz Fernando Cótica

do)'https://doi.org/10.37572/EdArt_20122152116

CAPÍTULO 17.

EFEITO DO COBALTO E ZINCO EM VARISTORES À BASE DE SNO

Glauco Meireles Mascarenhas Morandi Lustosa

João Paulo de Campos da Costa

Leinig Antônio Perazzoli

Biljana Stojanovic 
Maria Aparecida Zaghete Bertochi

Elson Longo

doi)'https://doi.org/10.37572/EdArt_20122152117

CAPÍTULO 18 250

GRADUAÇÃO HISTOLÓGICA DOS GLIOMAS PELA ANÁLISE DA PERMEABILIDADE MICROVASCULAR POR RESSONÂNCIA MAGNÉTICA

Pedro Henrique Raffa de Souza

Rodrigo de Oliveira Plotze

Lucas Giansante Abud

Carolina Baraldi Araújo Restini

do)' https://doi.org/10.37572/EdArt_20122152118

CAPÍTULO 19. 270

MONITORAMENTO DE ENCALHES DE ANIMAIS MARINHOS NA GESTÃO DA PESCA EM UNIDADES DE CONSERVAÇÃO

Viviane Korres Bisch

Roberto Sforza

do)'https://doi.org/10.37572/EdArt_20122152119

SOBRE O ORGANIZADOR. 280

ÍNDICE REMISSIVO 


\section{CAPÍTULO 19}

\section{MONITORAMENTO DE ENCALHES DE ANIMAIS MARINHOS NA GESTÃO DA PESCA EM UNIDADES DE CONSERVAÇÃO}

Data de submissão: 24/09/2021

Data de aceite: 14/10/2021

Viviane Korres Bisch Universidade Federal do Paraná Centro de Estudos do Mar

Pontal do Sul - PR http://lattes.cnpq.br/2732917756160901

Roberto Sforza

NGI ICMBio Santa Cruz

Vitória - ES

http://lattes.cnpq.br/0275630136552061

RESUMO: Esse trabalho aplicou uma ferramenta de Sistema de Informações Geográficas para análise espaço-temporal de ocorrências de encalhes de quelônios marinhos relacionados à interação com atividade pesqueira nas praias das Unidades de Conservação (UCs) Área de Proteção Ambiental (APA) Costa das Algas e do Refúgio de Vida Silvestre (RVS) de Santa Cruz (Brasil), as quais são diariamente monitoradas pelo Programa de Monitoramento de Praias da Bacia de Campos e do Espírito Santo PMP BC/ES. Para análise dos resultados, gráficos foram gerados no Excel e mapas produzidos no software ArcGis (ArcMap 10). Os resultados indicam que a região costeira das UCs apresentaram encalhes devidos a interação com pesca, avaliados pelas marcas e materiais de pesca encontrados nos organismos encalhados, mostrando que, no período observado, o setor referente à localidade das UCs apresentou mais que o dobro do somatório de encalhes no setor ao norte e ao Sul do mesmo. Além disso, o número de ocorrências de encalhe por interação com a pesca também demonstrou um aumento significativo ao longo dos anos, saindo de três em 2010 e aumentando gradativamente até alcançar seu máximo registrado em 2017, com 173 registros de encalhe devido à interação com pesca. O maior número de encalhes é representado pela espécie Chelonia Mydas (95\%). Uma vez verificado o impacto que a pesca de rede em áreas de lateritas tem na população de quelônios, levando os indivíduos afetados à óbito, se sugerem medidas para uma interação sustentável entre a pesca tradicional e a conservação da biodiversidade.

PALAVRAS-CHAVE: Unidades de Conservação. SIG. Quelônios Marinhos.

\section{MONITORING OF MARINE TURTLE STRANDINGS AND COASTAL MANAGEMENT IN CONSERVATIONS UNITS}

ABSTRACT: This work applied a Geographic Information System (GIS) tool for analysis of spatial-temporal occurrences of strandings of marine turtles related to the interaction fishing 
on the beaches of APA Costa das Algae and RVS Santa Cruz. The paper uses data collected by the Campos Basin Beach Monitoring Program and Espírito Santo - PMP BC / $E S$, which constitutes a program of monitoring of marine animals on the beaches of these sedimentary basins, to assess possible interactions with oil and gas exploration, production and disposal activities, such as environmental licensing processes of these activities. The results indicate that the coastal region of the Protected Areas (PA) presents beaching by interaction with fishing, especially net fishing, which is used mainly in areas with lateritic armor in the region. Besides the the number of occurrences of these strandings is higher in the area of the mentioned units of than in the southern and northern monitored regions of these PAs, the number of occurrences of beaching by interaction with fishing also demonstrated an increase in over the years.

KEYWORDS: Conservation Units. GIS. Marine Turtles.

\section{INTRODUÇÃO}

Na região costeira do município de Aracruz encontram-se terraços ou plataformas de abrasão da Formação Barreiras, formados por concreções lateríticas, chamados também de couraças lateríticas ou, simplesmente, lateritas. Esses blocos formam um substrato rochoso e extremamente poroso e fragmentado, que até $600 \mathrm{~m}$ do mediolitoral podem chegar a ficar descobertos na baixa mar, onde permanecem então poças de maré. A importância ecológica da presença dessas plataformas é demonstrada em estudos da região, onde destaca-se o papel de agregação de algas e subsequente recrutamento de animais para a região (Longo, 1997 e Maioli, 2009).

O hábito costeiro de quelônios, em especial a tartaruga verde (Chelonia mydas) que são atraídos para regiões tais como as couraças lateríticas, os tornam suscetíveis à interação com a pesca costeira, principalmente a de rede de emalhe, atingindo diretamente a população de juvenis (Sales et al., 2008, Bugoni et al., 2003).

Quelônios marinhos são reconhecidos por seu papel ecológico e, nos últimos anos, as pesquisas e projetos no ramo da conservação destes animais apresentou um grande incremento, uma vez que foi constatado o risco de extinção destes animais, principalmente no Brasil. Atualmente, esse táxon é protegido por leis ambientais, sendo o Brasil um dos países signatários da Convenção sobre Comércio Internacional de Espécies Ameaçadas da Flora e Fauna Silvestres de Extinção - CITES e da IAC, Convenção Interamericana para a Proteção e Conservação das Tartarugas Marinhas (Almeida, 2011).

Estudos como Reisser (2003) corroboram para a compreensão de que a pesca realizada por comunidades de pescadores é a principal causa de óbito de quelônios marinhos e, na região de Aracruz, Netto (2002) e Assis (2016) confirmam que poluição não é diretamente relacionada ao óbito destes animais, e sim a interferência humana, caracterizada por grande atividade de pesca, inclusive artesanal. 
Almeida (2011) sugere que, para manutenção do estado de conservação desses animais, é fundamental a implantação de Unidades de Conservação marinhas e costeiras, realizar estudos para levantamento da interação humana com o táxon e estimular conexão com comunidades pesqueiras para a melhor alternativa viável e ordenamento.

$\mathrm{Na}$ área de estudo destaca-se a presença de Unidades de Conservação (UC) Federais de núcleo integrado de gestão, a Área de Proteção Ambiental (APA) Costa das Algas e o Refúgio de Vida Silvestre (RVS) de Santa Cruz. A APA localiza-se entre os municípios de Serra, Fundão e Aracruz e é classificada como unidade de conservação de uso sustentável, de forma que entre seus objetivos constam proteger a diversidade biológica e os ambientes naturais, principalmente os fundos colonizados por algas, garantir a valorização das atividades pesqueiras e extrativistas de subsistência e realizar ordenamento do uso dos recursos naturais pesqueiros e demais organismos marinhos. O RVS é uma unidade de conservação de proteção integral, cujos objetivos contam são proteger a diversidade biológica e os ambientes naturais, contribuir para a recuperação dos recursos biológicos e para a sustentabilidade das atividades pesqueiras e extrativistas de subsistência e de pequena escala praticadas pelas comunidades costeiras da região no entorno da Unidade de Conservação, entre outros (Decreto de 17 de junho de 2010).

A fim de cumprir seus objetivos, UCs devem organizar seu Plano de Manejo, documento no qual são definidas as áreas destinadas à proteção integral dos ecossistemas e aquelas onde os recursos biológicos estão passíveis de utilização para atividades pesqueiras, sendo que a arte de pesca também deve ser considerada de forma a serem compatíveis com os objetivos de conservação da UC (Decreto de 17 de junho de 2010).

Nesse contexto, existem ferramentas que podem auxiliar no entendimento dessa interação antrópica com o encalhe e óbito de animais marinhos que estão em risco, tal como o Projeto de Monitoramento de Praias das Bacias de Campos e do Espírito Santo PMP-BC/ES, que constitui programa de aquisição de dados e confecção de relatórios a respeito de animais encontrados encalhados em praias das bacias de Campos e Espírito Santo. Abrange uma extensão de, aproximadamente, $763 \mathrm{~km}$ de linha de praia, os quais se localizam entre o limite norte do município de Conceição da Barra, no Espírito Santo, e o limite sul do município de Saquarema, no Rio de Janeiro.

O intuito do presente estudo é avaliar se há evidência de interação da pesca com rede como causador de mortalidade de quelônios e se essa interação está ligada à presença das couraças lateríticas. Para tal, a ferramenta de comparação dos resultados espacialmente por SIG se torna uma ferramenta importante para a visualização da área a ser analisada e onde estão as ocorrências. O trabalho corrobora, dessa forma, com a validação da importância de projetos como o PMP na geração de informações que subsidiam a gestão de Unidades de Conservação. 


\section{METODOLOGIA}

Foi feita a análise do $7^{\circ}$ Relatório Anual do Projeto de Monitoramento de Praias das Bacias de Campos e do Espírito Santo - PMP-BC/ES, consolidando os resultados desde $1^{\circ}$ de outubro de 2010 até 30 de setembro de 2017, englobando os sete anos consecutivos da sua execução.

Das 14 áreas que o PMP engloba estabeleceu-se que as áreas de interesse do monitoramento para as Unidades de Conservação (UCs) Área de Proteção Ambiental (APA) Costa das Algas e Refúgio de Vida Silvestre (RVS) de Santa Cruz são as áreas 2B - Linhares e Aracruz (sul da foz do Rio Doce até a foz do rio Riacho-Comboios), 3A - Aracruz e Fundão (sul da foz do Rio Riacho), e 3B - Serra, Vitória e Vila Velha, visto que, devido à proximidade geográfica, essas áreas podem causar influência nos registros dentro das Unidades, além de também sofrerem a influência das UCs.

Para análise dos resultados, gráficos foram gerados através da sistematização dos dados brutos de interesse em tabelas separadas da planilha original, de forma a filtrar as informações consideradas relevantes para o foco do trabalho.

Para as ocorrências não reprodutivas (encalhes) de tartarugas marinhas, as informações consideradas importantes foram data da ocorrência (ano), município e praia, sendo que esses geram como produtos os gráficos da distribuição temporal e distribuição espacial.

É importante ressaltar que, por ser um projeto de ampla extensão e ampla mobilização de funcionários, o PMP pode contar com pequenas discrepâncias como, no caso, a nomenclatura utilizada no preenchimento das fichas no momento de identificação das evidências que o quelônio esteve em contato com a pesca. Dessa forma, o que alguns preencher como "Sim" outros teriam preenchido como "Rede", entre outros. Mesmo sujeito à melhorias, os dados são muito úteis para avaliação do panorama geral das ocorrências.

Para a criação dos mapas (Figura 1 e 2) foi utilizado também filtro nos dados com foco no critério chamado de interação com a pesca, o qual foi transformado, junto com as coordenadas geográficas, em arquivo de formato .cvs que foi introduzido no software ArcGis (ArcMap 10). No software, então, foi transformado em arquivo shapefile e, a partir de filtro na tabela de atributos, foram criados vários shapefiles, cada um contendo os pontos referentes a um tipo de interação com a pesca. A partir desses dados foram organizados os mapas.

\section{RESULTADOS}

A região de estudo compreende a área costeira entre os município de Vila Velha e Linhares, no Estado do Espírito Santo (Figura 1). 
$\mathrm{Na}$ área 3A, de maior foco do estudo, a ocorrência de couraças lateríticas propicia o crescimento de algas por propiciar um substrato rochoso e isso acaba por atrair tartarugas verdes (Chelonia mydas) por se alimentarem de algas verdes na fase juvenil e sua distribuição se estender na costa do Espírito Santo (Proietti et al. 2009).

O litoral do Espírito Santo, em sua porção mais norte, representa um importante sítio reprodutivo para as tartarugas cabeçudas (Caretta caretta) e outros pontos de desova ocorrem em menor quantidade ao longo de todo o litoral do estado (Marcovaldi \& Chaloupka, 2007).

Figura 1 - Mapa da Área de Estudo, em evidência os pontos de divisa entre as áreas 2B, 3A e 3B, as Unidades de Conservação e as ocorrências de couraças lateríticas.

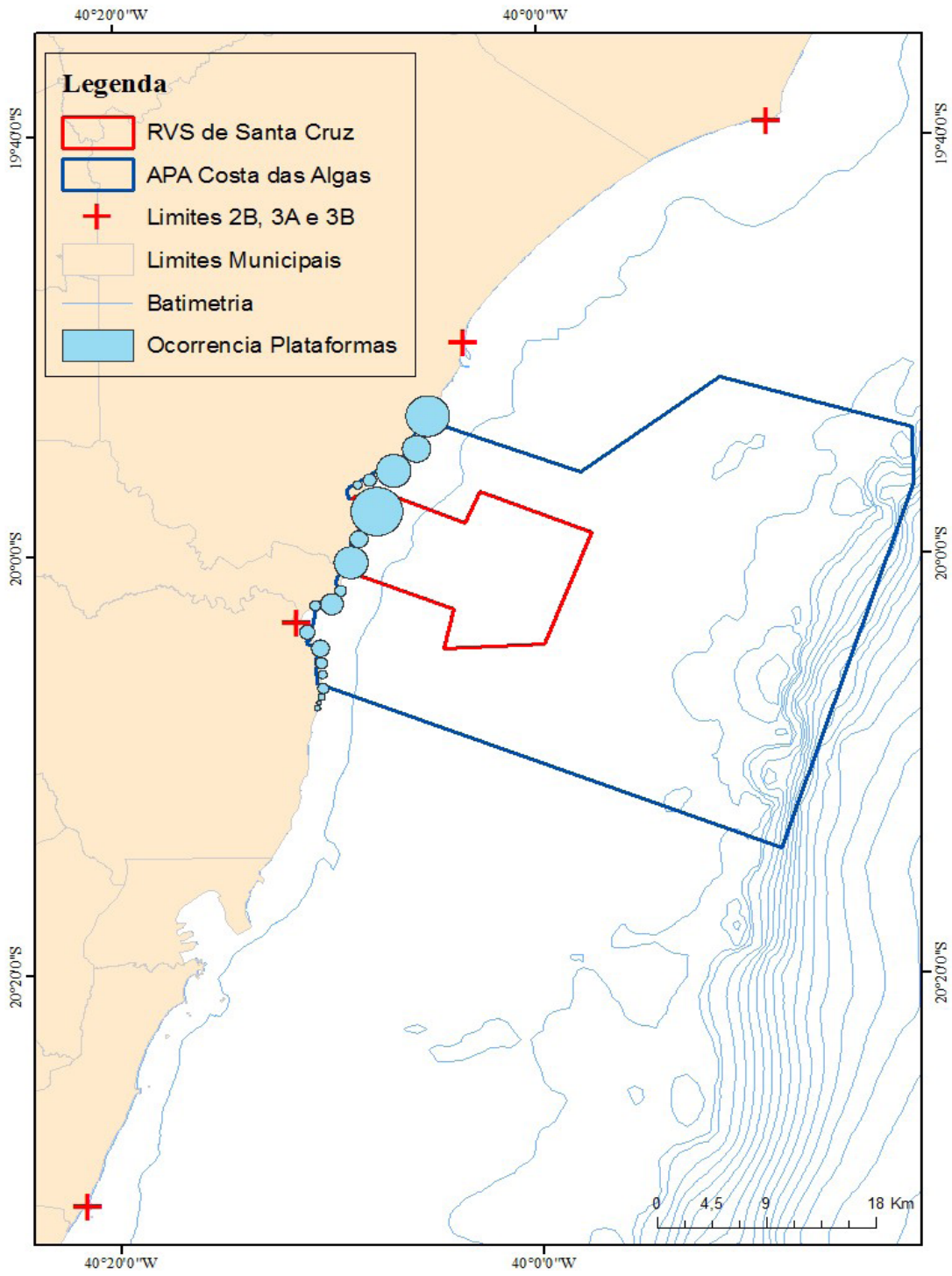


Fig 2 - Ocorrências de encalhe de quelônios marinhos com a determinação do tipo de interação com a pesca (a) Sim, (b) Forte, (c) Total e (d) Rede.
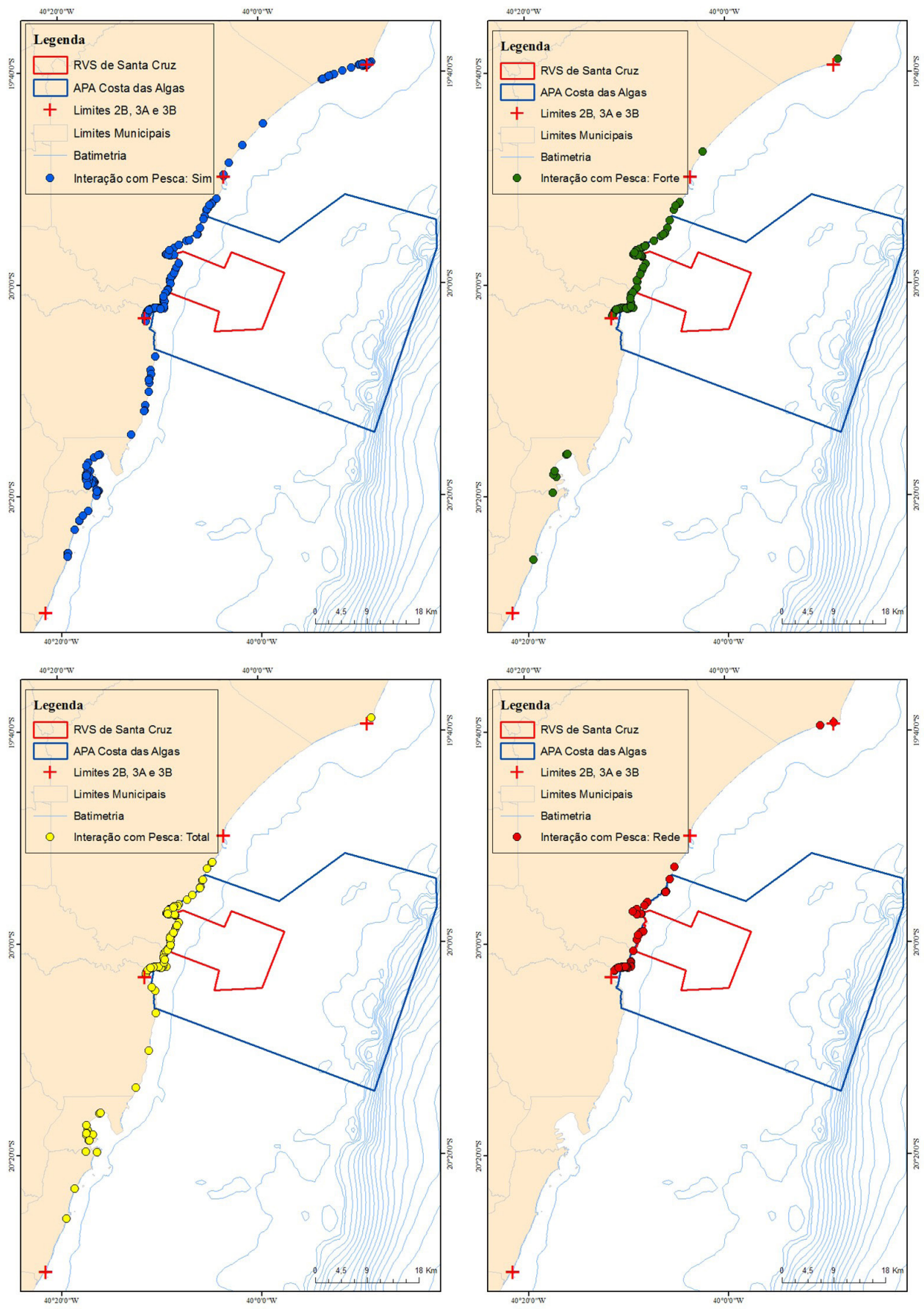

Nos mapas (Figura 2), é perceptível que, à medida que o nível de detalhamento da interação com atividade de pesca e o nivel de interação, as ocorrências começam a se 
concentrar na área referente à região costeira das unidades de conservação, na qual se tem a presença das couraças lateríticas.

Fig 3 - Distribuição espacial de tipo de ocorrência de interação com pesca durante todos os anos de monitoramento do PMP-BC/ES.

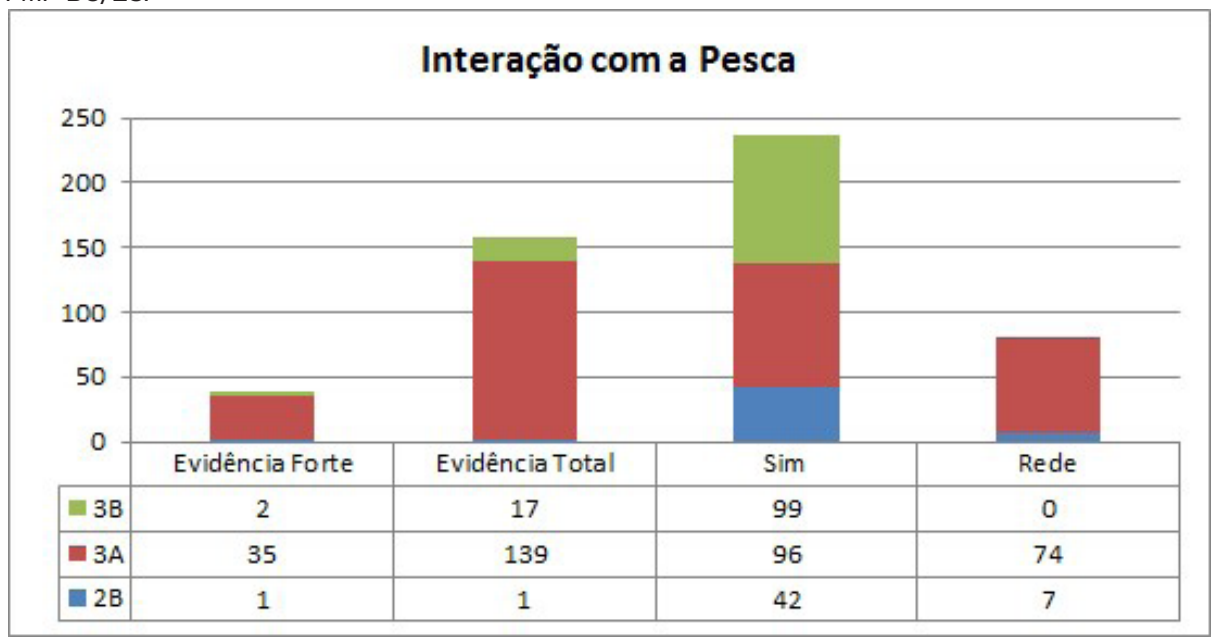

Fig 4 - Distribuição temporal dos encalhes de quelônios relacionados à pesca durante todos os anos de monitoramento do PMP-BC/ES.

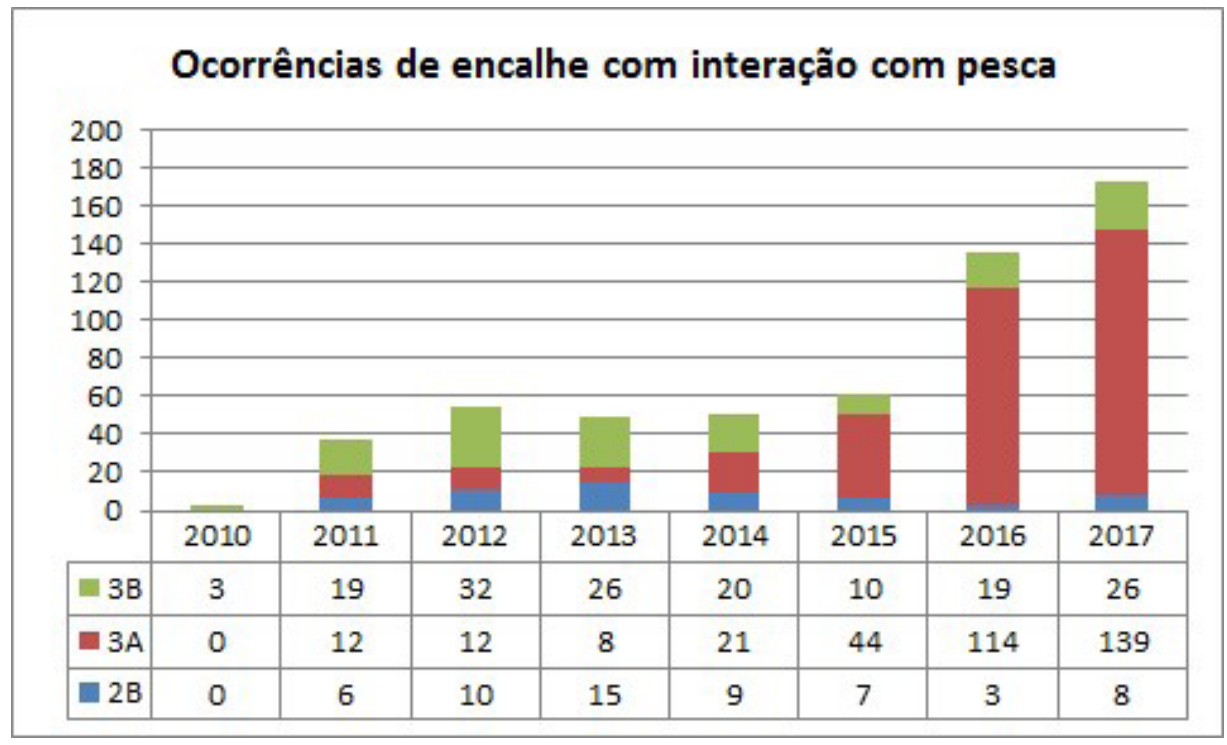

Os resultados mostram que, de fato, os registros que contém definição da pesca de rede como causa direta de encalhes de quelônios estão, em sua maior parte, na área $3 \mathrm{~A}$, na região que compreende a faixa predominante de couraças lateríticas, região costeira das Unidades de Conservação federais. 
Outro fato que a análise de dados realça é que, das ocorrências de encalhe, 95\% são de tartarugas da espécies Chelonia mydas, a tartaruga verde, o que condiz com o fato de a região costeira ser região de alimentação destes animais. A espécie com segundo maior índice de ocorrência foi a Caretta caretta, em conformidade com o fato de a área de estudo apresentar vários pontos de desova dessa espécie registrados pelo Tamar.

Observa-se na Figura 4 que durante os anos de monitoramento do PMP houve um aumento exponencial e muito significativo na quantidade de indivíduos encontrados encalhados com evidência de interação com a pesca. Esse aumento se torna novamente evidente na área 3A, ou seja, na área onde estão incluídas as UCs e as lateritas.

Nos últimos 2 anos de monitoramento houveram as maiores quantidades de ocorrências de encalhe devido à interação com atividade pesqueira, demonstrando uma maior urgência de tomada de decisão com relação à atividade, uma vez que ela está afetando a biodiversidade da região.

\section{DISCUSSÃO E CONCLUSÃO}

A análise se mostrou eficiente para a identificação de uma relação forte entre a pesca de rede e a presença das couraças lateríticas na região das Unidades de Conservação estudadas. Esse resultado se mostra com importante necessidade de divulgação de forma que os órgãos ambientais se tornem cientes da problemática da região e possam, com isso, subsidiar as ações, tais como avaliar a proibição da arte de pesca do tipo rede, que traz impacto ambiental grande comprovado e a recomendação da substituição por outra e considerar a forma heterogênea do local, presente nas lateritas, no plano de manejo dos recursos e biodiversidade da região.

A grande mortalidade comprovada de Chelonia mydas parece, então, estar diretamente ligada ao processo de, ao virem se alimentar na região das lateritas, onde há acúmulo de algas no substrato rochoso, as tartarugas encontram um habitat favorável para permanecer em sua fase juvenil. Porém, a pesca de rede, na qual o pescador deixa a rede para posterior recuperação, prende as tartarugas de forma que impossibilita a subida para respirar ou impede sua liberação da rede, podendo ficar sem condições de capturar o alimento ou ficar expostas ao sol e sofrer ressecamento, levando eventualmente o animal a óbito.

Compondo a maioria dos registros, é essencial tomar medidas mitigadoras para amenizar o impacto sobre a tartaruga verde, uma vez que a mesma já se encontra classificada como espécie vulnerável, segundo Perez et al. (2011), avaliando pelos critérios da IUCN (2001). Almeida et al. (2011) já alertam que a tartaruga verde é a que possui maior número de encalhes em toda a região costeira devido à pesca de emalhe. 
A comprovação da crescente mortalidade de quelônios devido à pesca e a identificação espacial de onde o problema ocorre é fundamental a construção do plano de manejo das referidas UCs, uma vez que as mesmas ainda não o possuem, de forma que se possam implantar as políticas necessárias para a manutenção da biodiversidade e que a atividade de pesca se torne sustentável.

Os dados de PMP apresentam algumas lacunas como ter monitoramento conjunto com o tamar em algumas áreas e não ter nas outras, a incerteza sobre o controle do trabalho de campo devido a possíveis divergências de interpretação do responsável por registrar a ocorrência. Entretanto, o PMP se mostrou relevante para o entendimento de processos em área de interesse de preservação ambiental e gestão sustentável entre a biodiversidade e a atividade humana.

Este trabalho é inovador no quesito de se utilizar análise espacial para a visualização do problema em questão, permitindo uma compreensão mais fácil de como ocorre o problema, o que foi corroborado pelos gráficos. Sendo assim, a sua replicação é possível e interessante, uma vez que dados de PMP são públicas o programa ArcGis possui um similar, o QGis, que é uma plataforma gratuita, podendo ser utilizado para analisar diferentes questões a serem investigadas.

\section{REFERÊNCIAS}

ALMEIDA, A. P., SANTOS, A. J. B., THOMÉ, J. C. A., BELINI, C., BAPTISTOTTE, C., MARCOVALDI, M. Â., Lopez, M. (2011). Avaliação do estado de conservação da tartaruga marinha Chelonia mydas (Linnaeus, 1758) no Brasil. Biodiversidade Brasileira, (1).

ASSIS, F. D. P. G., \& CALDARA, S. R. L. (2016). Causa de óbitos de tartarugas marinhas das praias de Aracruz-ES, Brasil.

LONGO, L.L. 1997. Repartição espaço temporal da cnidofauna em arenito de praia em Santa Cruz (Aracruz-ES). Instituto de Biociências, São Paulo.

MAIOLI, D. G. (2009). Composição e abundância da meiofauna bentônica de rodolitos e sedimentos depositados nos recifes de couraças lateríticas de Santa Cruz, Aracruz-ES. Vitória. Monografia (Bacharelado em Oceanografia e Ecologia), Universidade Federal do Espírito Santo. 43p.

MARCOVALDI, M.A. \& CHALOUPKA, M. Conservation status of the loggerhead sea turtle in Brazil: an encouraging outlook. Endangered Species Research, 3: 133-143.

NETTO, R. F., NUNES, A. G. A., \& ALBINO, J. (2002). A pesca realizada na comunidade de pescadores artesanais de Santa Cruz/ES-Brasil. Boletim do Instituto de Pesca, São Paulo, 28(1), 93-100.

PROIETTI, M. C.; LARA-RUIZ, P.; REISSER, J. W.; PINTO, L. S.; DELLAGOSTIN, O. A.; MARINS, L. F. 2009. Green turtles (Chelonia mydas) foraging at Arvoredo Island in Southern Brazil: Genetic characterization and mixed stock analysis through mtDNA control region haplotypes. Genetics and Molecular Biology, 32(3): 613-618. 
IUCN. 2001. IUCN Red List Categories and Criteria: Version 3.1. IUCN Species Survival Commission, IUCN. 30p.

PERES, M.B.; DIAS, B.F.S. \& VERCILLO, U.E. 2011. Avaliação do estado de conservação da fauna brasileira e a lista de espécies ameaçadas: $O$ que significa? Qual sua importância? Como fazer? Biodiversidade Brasileira, 1: 45-48.

REISSER, J. W; PROIETTI, M. C. \& KINAS, P. G. 2003. Tartarugas marinhas da ilha do arvoredo, reserva biológica marinha do arvoredo, SC. In: Livro de Resumos da II Jornada de Conservação e Pesquisa de Tartarugas Marinhas no Atlântico Sul Ocidental. NEMA/FURG. Rio Grande do Sul - Brasil. 


\section{SOBRE O ORGANIZADOR}

Leinig Antonio Perazolli possui graduação em Engenharia Química pela Universidade Estadual de Maringá (1986), mestrado em Engenharia Química pela Universidade Estadual de Campinas (1991) e doutorado em Química pela Universidade Federal de São Carlos (1996). Atualmente é professor Livre Docente III do Instituto de Química - Unesp / Araraquara. Tem experiência na área de Engenharia de Materiais e Metalúrgica com estudos de Sinterização de Cerâmicos e obtenção de Foto catalisadores Cerâmicos e na área de História da Ciência com ênfase em Química e Engenharia Química. Atua na área de pesquisa nos seguintes temas: óxido de titânio, óxido de estanho, sinterização, voltados para a foto catálise e cerâmicas eletrônicas. $\mathrm{Na}$ área de extensão universitária desenvolve trabalhos sobre História da Ciência e da Engenharia Química e sobre a Química das Coisas. Leciona disciplinas na área de Química Tecnológica, Engenharia Química e História da Ciência. 


\section{ÍNDICE REMISSIVO}

A

Absorción 106, 107, 110, 142, 171, 176, 178, 180, 181, 182, 183, 184, 188

Aceite $147,148,149,150,151,153,154,155,156,157,158,159,160,161,162,163,164,165$, $166,167,169$

Aguacate $147,148,149,150,151,153,154,155,156,157,158$

Aislación térmica 114, 115, 117, 120, 131, 132

Análisis 1, 2, 5, 7, 8, 9, 10, 13, 14, 16, 20, 23, 24, 26, 30, 32, 69, 86, 87, 89, 90, 95, 116, 129, $131,134,136,137,138,139,141,142,144,162,163,167,173,186,188,189$

Antimicrobiana 134, 135, 136, 148, 158, 161, 169

Apatita $186,187,188,189,191$

Aplicaciones 43, 160

A-site substitutions 227

Aspergillus niger 103, 104, 105, 111, 112, 113

Avaliação 47, 58, 59, 63, 64, 66, 67, 68, 250, 253, 255, 256, 257, 258, 259, 260, 261, 262, $264,265,268,273,278,279$

B

$\mathrm{BiFeO}_{3} 227,228,231,232,233$

Biomasa 103, 104, 106, 107, 108, 109, 110, 111, 113, 160

C

Climas cálidos 114, 117, 129, 130

Cohesión social 69

Combustiveis renováveis 214

Composición proximal 160, 168

Compresión 121, 171, 172, 173, 174, 175, 182, 183, 184

Consumidor ético 1, 2, 7, 11, 14

Consumo energético 114, 115, 116, 117, 122, 123, 124, 125, 126, 127, 128, 129, 130, 131, 217

Consumo ético 1, 2, 3, 5, 6, 7, 8, 9, 10, 11, 14, 15, 16

Consumo responsable 1

Contaminación Difusa 18, 19, 22, 33

Control interno 89, 92, 93, 94, 95, 96, 97, 98, 99, 100, 101, 102

Cuprita $134,136,137,138,139,140,141,142,143,144,145$ 
D

Densidad 166, 171, 175, 177, 179, 180, 182, 183, 193, 197, 206, 207, 211, 238, 239, 243, 245, 246

Deposição eletroforética 234, 235, 237, 240, 248

Desarrollo 8, 18, 20, 21, 32, 37, 39, 40, 41, 42, 43, 46, 69, 70, 75, 76, 77, 84, 85, 86, 87, 88, $90,91,102,149,153,154,155,167,170,171,172,176,179,187$

Difusão 207, 234, 235, 241, 243, 245, 254

Difusão de Cromo 235

$E$

Ecosistemas de Emprendimiento 36, 37, 38

Emancipatória 47, 58, 59, 64, 66, 68

Emprendimiento $36,37,38,39,40,41,42,43,44,45$

Espectroscopia 200, 251, 253, 268

Etanol 214, 215, 216, 217, 221, 222, 224, 225

Ética del consumo 1

Extração líquido-líquido 214, 218, 221, 222, 223, 224, 225

$\mathbf{F}$

Ferric properties 227

Flotación 186, 187, 188, 189, 190, 191

G

Glioma 250, 251, 252, 253, 254, 267, 268, 269

Glioma Astrocítico 251

I

Imagem de Perfusão 251

M

Materiais compósitos com matriz de alumínio 193, 194

Metales pesados 103, 104, 105, 106, 107, 108, 109, 110, 111

Moringa oleifera Lam 159, 160, 161, 162, 163, 164, 165, 167, 168, 169

Municipios locales 89 
Normas $1,5,6,7,8,13,14,15,89,95,97,100,102$

O

Óxido 109, 134, 135, 136, 137, 145, 146, 196, 235

Óxido de estanho 235

$\mathbf{P}$

Percepción $6,8,9,10,11,14,15,69,70,71,74,75,76,79,81,82,83,84,87,88$

Perfusão 251, 253, 254, 255, 256, 258, 259, 261, 264, 265, 267, 268

Permeabilidade Capilar 251

Porosidad 171, 176, 177, 178, 180, 181, 182, 183, 184

Potencialidades 65, 147, 148, 149, 169

Procedimiento LU-IV 18, 19, 20, 21, 23, 27, 28, 29, 30, 31, 32

Q

Quelônios marinhos 270, 271, 275

$\mathbf{R}$

Relave 186, 188, 189, 191, 192

Remoción 98, 100, 103, 104, 105, 106, 107, 108, 109, 110

Resíduos de mineração 194

Ressonância Magnética 250, 251, 253, 254, 268

Roca $171,172,173,174,175,176,177,178,182,183,184$

S

Santander 36, 37, 41, 42, 43, 44, 45, 46, 186

Semillas 147, 155, 159, 160, 161, 162, 164, 169

SIG 18, 20, 270, 272

Sinerização Microondas Varistores 235

Sinterização 193, 194, 196, 197, 206, 207, 208, 210, 211, 234, 236, 237, 240, 241, 243, 244 , 245, 246, 249

Sistemas de Información Geográfica 19, 20

Staphylococcus aureus 134, 135, 136, 142, 144, 145, 146 
Tecnologias 47, 49, 52, 53, 57, 59, 60, 67, 68, 79, 83, 120, 145, 184

$\mathbf{U}$

Unidades de Conservação 270, 272, 273, 274, 276, 277

UNIFAC 214, 215, 218, 219, 221

Universidad compleja 69,88

Z

Zonas Vulnerables a la Contaminación por Nitrato (ZVN) 18, 19 